\title{
Evaluación fisicoquímica y biológica en sistemas de tratamiento comerciales en Costa Rica
} Physical-chemical and biological evaluation in
commercial treatment systems in Costa Rica

Guillermo Calvo-Brenes ${ }^{1}$, Diana Zambrano², Sofía Picado-Valverde ${ }^{3}$

Calvo-Brenes, G; Zambrano, D; Picado-Valverde, S. Evaluación fisicoquímica y biológica en sistemas de tratamiento comerciales en Costa Rica. Tecnología en Marcha. Vol. 33-4.

Octubre-Diciembre 2020. Pág 47-61.

doi) https://doi.org/10.18845/tm.v33i4.4496

1 Centro de Investigación en Protección Ambiental (CIPA), Escuela de Química, Instituto Tecnológico de Costa Rica. Costa Rica. Correo electrónico: gcalvo@tec.ac.cr.

2 Centro de Investigación en Protección Ambiental (CIPA), Escuela de Química, Instituto Tecnológico de Costa Rica. Costa Rica. Correo electrónico: dzambrano@tec.ac.cr.

3 Centro de Investigación en Vivienda y Construcción (CIVCO), Escuela de Ingeniería de la Construcción, Instituto Tecnológico de Costa Rica, Costa Rica. Correo electrónico: sapicado@tec.ac.cr. 


\title{
Palabras clave
}

Aguas residuales domésticas; sistemas de tratamiento; tanques sépticos; filtro anaerobio; evaluación físico-química; evaluación biológica; evaluación hidrodinámica.

\section{Resumen}

En Costa Rica, el 70.9\% de la población utiliza el tanque séptico como sistema de tratamiento individual a las aguas residuales domésticas. A pesar de que los tanques sépticos son la tecnología de tratamiento más utilizada en el país, no se cuenta con información detallada acerca de su funcionamiento y su eficiencia. Además, labores de mantenimiento, como lo es la remoción de lodos sépticos, son ejecutadas directamente por cada familia que usa este sistema y sin que exista un protocolo de limpieza unificado. La evaluación de estos sistemas se llevó a cabo en sistemas de tratamiento fabricados por una de las empresas privadas más importantes del país. Cada equipo evaluado consistía en un tanque séptico y un filtro anaerobio de flujo ascendente (FAFA) para el tratamiento de aguas residuales domésticas. La caracterización incluyó aspectos físicos, químicos y biológicos de la calidad y cantidad del agua residual producida en tres sistemas de tratamiento distintos, considerando variaciones horarias, así como la calidad y la cantidad de lodos acumulados en cada sistema. Los sistemas de tratamiento estaban instalados en dos edificios multifamiliares y una casa unifamiliar. Los resultados indicaron que los sistemas de tratamiento son muy sensibles en cuanto a cantidad y calidad del agua residual, presentando variaciones horarias significativas y además, afectadas por el tipo de uso de agua que se da en los hogares; lo cual está directamente relacionado al funcionamiento del tratamiento.

\section{Keywords}

Domestic wastewater; treatment systems; septic tanks; anaerobic filter; physical-chemical evaluation; biological evaluation; hydrodynamic evaluation.

\begin{abstract}
In Costa Rica, $70.9 \%$ of the population uses the septic tank as an individual treatment system for domestic wastewater. Although septic tanks are the most used treatment technology in the country, there is no detailed information about their operation and efficiency. The maintenance tasks, such as the removal of septic sludge, was carried out directly by each family that uses this system, using their own resources. The evaluation was carried out in treatment systems manufactured by one of the most important private companies in the country. Each equipment consists of a septic tank and an upflow anaerobic filter for the treatment of domestic wastewater. The evaluation included a physical, chemical and biological characterization of the quality and quantity of wastewater produced in three different treatment systems, considering hourly variations, as well as the characterization of the quality and quantity of sludge accumulated in each system. The treatment systems were installed in two multifamily buildings and a singlefamily house. The results indicated that the treatment systems are very sensitive in terms of quantity and quality of wastewater, presenting significant hourly variations and also affected by the type of water use that occurs in homes; which is directly related to the operation of the treatment.
\end{abstract}




\section{Introducción}

En Costa Rica, el 70,9\% de la población utiliza el tanque séptico como sistema de tratamiento para sus aguas residuales domésticas. El tanque séptico es un sistema anaerobio capaz de tratar aguas residuales domésticas, institucionales y en algunos casos industriales; a partir de un proceso físico, químico y biológico [1]. Sin embargo, existen grandes deficiencias y aspectos que no se han evaluado tanto en diseño como en operación, mantenimiento y función de estos sistemas de tratamiento [2]. Las labores de mantenimiento, como lo es la remoción de lodos sépticos, son ejecutadas directamente por cada familia que usa tanque séptico, convirtiéndose en un servicio privado y no regulado por ningún ente estatal. Además, la regulación y gestión legal en el país es desactualizada y limitada, lo cual implica un manejo que no es totalmente adecuado, impactando los cuerpos de agua superficial e incrementando el riesgo de contaminación de las aguas subterráneas.

La caracterización del agua residual es indispensable en el diseño de un sistema de depuración de contaminantes, por lo que saber la calidad de los parámetros físicos y químicos es uno de los primeros pasos al tratar con aguas residuales [3]. La temperatura y el pH son dos variables físicas muy importantes, ya que afectan directamente el crecimiento de microorganismos y por lo tanto, el adecuado funcionamiento biológico del sistema. Otra característica importante, son los sólidos suspendidos totales (SST), que corresponde a aquellas partículas flotantes en el agua y que pueden ser separados del medio líquido a partir de tratamientos físicos sencillos o por medios mecánicos. La naturaleza de los sólidos suspendidos totales es principalmente orgánica [4]. Uno de los indicadores de materia orgánica más importantes es la Demanda Bioquímica de Oxígeno $\left(\mathrm{DBO}_{5}\right)$ que corresponde a la determinación de la cantidad de oxígeno disuelto que consumen los microorganismos en el proceso de oxidación de materia orgánica biodegradable en un lapso de tiempo de cinco días a $20^{\circ} \mathrm{C}$ [5].

Se reporta en la literatura valores de entrada al sistema en SST que van desde los 100 hasta los 400 mg/L y en el caso del $\mathrm{DBO}_{5}$ este puede variar de $250 \mathrm{mg} / \mathrm{L}$ hasta los 800 [6]. Los parámetros varían según el lugar o la comunidad de la que prevengan, por lo que los valores de un país desarrollado son diferentes a los de uno subdesarrollado, especialmente por la cantidad y la calidad del agua que se usa en estos sistemas. Productos de limpieza, higiene y hasta la dieta en las personas modifican la carga de las aguas residuales. En Estados Unidos, donde el

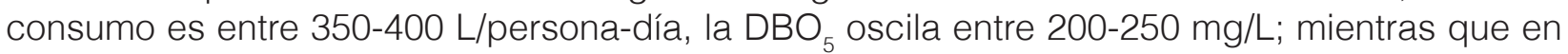
países tropicales subdesarrollados donde el consumo es la mitad o un cuarto de esa dotación la $\mathrm{DBO}_{5}$ es de 300-700mg/L [7]. El porcentaje de remoción en sólidos suspendidos podría estar aproximadamente en un rango entre 60 y $70 \%$.

Se considera que en países tropicales la remoción es mejor por la estabilidad en las temperaturas, las cuales son de 20 a $30^{\circ} \mathrm{C}$. Se ha reportado valores muy bajos en sistemas anaerobios como son los tanques sépticos y con temperaturas por debajo de los $10^{\circ} \mathrm{C}$; ascendiendo a un porcentaje entre $40-60 \%$ cuando las temperaturas son alrededor de los $20^{\circ} \mathrm{C}$. Una mejor eficiencia ocurre cuando las temperaturas del agua son mayores a los $25^{\circ} \mathrm{C}$ donde pueden obtenerse porcentajes de remoción de $\mathrm{DBO}_{5}$ hasta de 80\%. [8].

La estructura del tanque séptico es fundamental para un adecuado funcionamiento en la depuración de las aguas residuales. Desde la entrada hasta la salida, existen detalles que marcan la diferencia en la eficiencia del sistema. Teóricamente, se ha establecido que se puede utilizar la compartimentalización para tanques con volúmenes mayores a los 5 metros cúbicos, con un máximo de cuatro divisiones cada una de ellas con un largo mínimo de 0,60m. En el primer compartimento se llevaría a cabo el mayor porcentaje de sedimentación y digestión representando entre un 50-60\% de volumen de partículas sedimentada, dando lugar a la digestión más favorecida [9]. Debido a los procesos llevados a cabo por un reactor 
anaerobio, dentro del tanque séptico se da un almacenamiento de lodos en la parte inferior de la estructura; por la descomposición anaerobia y facultativa, el cual es hábitat para una gran cantidad de bacterias y microorganismos; las cuales tienen importancia ambiental por el efecto que podrían causar tanto dentro como fuera del tanque séptico [8].

Se han llevado a cabo diversas investigaciones con relación al uso de sistemas de tratamiento complementarios al del tanque séptico para mejorar la calidad del efluente. Una de estas alternativas es el uso de filtros anaerobios de flujo ascendente (FAFA) colocado a la salida del tanque séptico [10]. Este filtro está constituido por un tanque que contiene material de relleno de materia inerte que forma un lecho fijo y por lo general, son piedras en diferentes granulometrías que dan soporte a ese lecho. En ese relleno se lleva a cabo una fijación de microorganismos y los compuestos orgánicos solubles que no fueron removidos en la etapa de tratamiento anterior; estos compuestos son digeridos y convertidos en productos finales; en su mayoría gaseosos, como el metano o el dióxido de carbono [11]. Estudios llevados a cabo en Brasil por Busato y Palowsky [10] muestran los comportamientos y eficiencias de sistemas integrados en términos de materia orgánica y velocidades de remoción. Estos investigadores observaron que tanto el $\mathrm{pH}$ como la temperatura se mantienen constantes, mientras que la $\mathrm{DBO}_{5}$ presentó una remoción de la carga orgánica del 78\%, aproximadamente en todo el sistema compuesto, teniendo el mayor porcentaje de remoción en el reactor anaerobio (69\%) y en el caso de SST, se obtuvo una remoción general de un 73\%.

\section{Materiales y métodos}

\section{Descripción de las áreas de estudio}

El estudio se llevó a cabo en tres lugares: dos en edificios multifamiliares denominados en esta investigación como "Apartamentos" y otro llamado "Condominios". El tercero es una casa de habitación unifamiliar denominada como "Casa". La ubicación de los sitios de estudio se presenta en la figura 1 los cuales forman parte del cantón de Escazú ubicados en el Gran Área Metropolitana de Costa Rica.
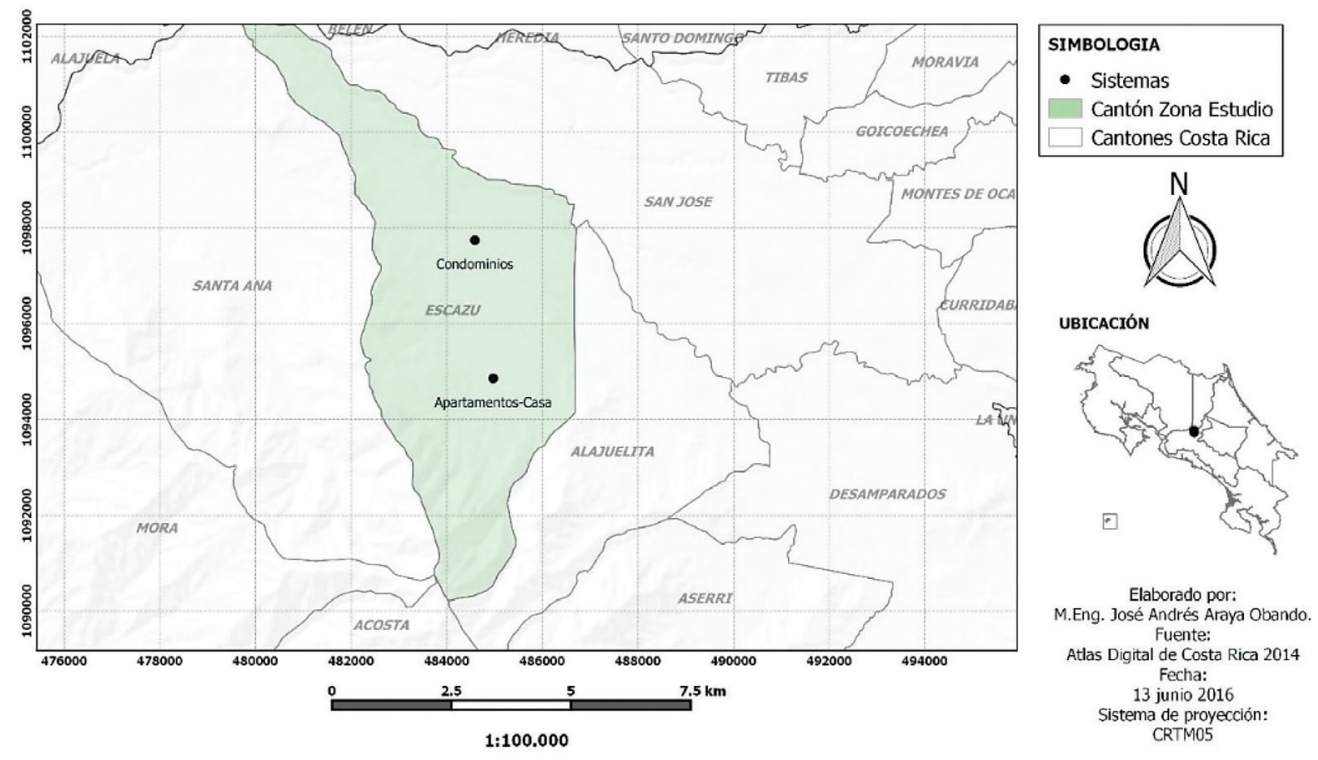

Figura 1. Ubicación de los sitios de estudio: Apartamentos, Condominios y Casa 
Los Apartamentos están compuestos por 4 unidades de vivienda, de nivel socioeconómico medio-alto, donde residen 10 personas en total. Durante el día son pocas las personas con permanencia en las residencias, ya que salen a sus actividades cotidianas, saliendo generalmente antes de las 9 de la mañana y regresando después de las 4 de la tarde. Las aguas residuales, están compuestas por la mezcla de aguas pardas y aguas grises. Las instalaciones hidrosanitarias de cada apartamento constan de 2 servicios sanitarios, 1 ducha, 2 lavatorios, un lavaplatos y una pila.

Los Condominios están conformados por 9 unidades de vivienda, de nivel socioeconómico alto y una casetilla de seguridad. En cada apartamento viven entre 1 ó 2 personas, teniendo actualmente un total de 14 residentes. La mayoría de las personas pasan fuera del condominio durante el día; por lo que aproximadamente sólo 6 ó 7 personas son las que permanecen en el edificio. Las aguas residuales, están compuestas por la mezcla de aguas pardas y aguas grises. Los aparatos hidrosanitarios por cada apartamento son, 2 servicios sanitarios, 2 lavatorios, 1 ducha, un lavaplatos, y una pila.

Por otra parte, el sitio Casa consta de una casa de habitación donde residen 6 personas y en la mañana, antes de las 7:00 a.m., la familia realiza las actividades rutinarias de aseo personal antes de salir a las ocupaciones cotidianas. Después, sólo se encuentra 1 persona dentro del hogar realizando las labores domésticas y durante la tarde se incorporan 3 personas más. Es decir, la casa está con su ocupación completa antes de las 7 de la mañana y después de las 5 de la tarde. Las aguas residuales generadas en el hogar son separadas respectivamente en sus tuberías, por lo que se analizaron únicamente las aguas pardas.

\section{Caracterización de las aguas residuales}

El levantamiento de los usos del agua y las características de las áreas de estudio se efectuó a partir de entrevistas a los encargados de cada uno de los lugares de estudio. La cuantificación de las de aguas residuales en los dos multifamiliares, se llevó a cabo a través de la medición del caudal por métodos volumétricos a lo largo de un día en lapsos de 15 a 30 minutos o cuando se presenciara un cambio en el flujo. En el sistema unifamiliar, no se levantaron caudales ya que estos están asociados con las descargas de los inodoros.

La caracterización en términos de calidad se realizó a partir de muestreos puntuales cada dos horas, entre el periodo de las 7:00 a.m. a 4:00 p.m. con una frecuencia mensual, durante 4 meses, los cuales se realizaban en la cámara de entrada de los respectivos sistemas de tratamiento. Tanto el $\mathrm{pH}$ como la temperatura se midieron en el sitio de ubicación de estos sistemas, mientras que los SST y la $\mathrm{DBO}_{5}$ se efectuaron en un laboratorio de análisis químico. Todos estos parámetros de calidad se evaluaron siguiendo la metodología establecida en el Standard Methods for the Examination of Water and Wastewater [12].

\section{Evaluación del desempeño de los sistemas de tratamiento}

En el tanque séptico de Apartamentos, se tomaron 3 puntos como referencia para los muestreos: uno en la entrada al tanque séptico, otro a la salida de éste y por último la salida del FAFA; tal y como se demarcan con equis en el diagrama de la figura 2.

En el sistema de tratamiento ubicado en los Condominios, se muestrearon 4 puntos: en la entrada del tanque séptico, en la salida de la cámara 1, salida de la cámara 3 y en la salida de los FAFA's; como se muestra en la figura 3. 


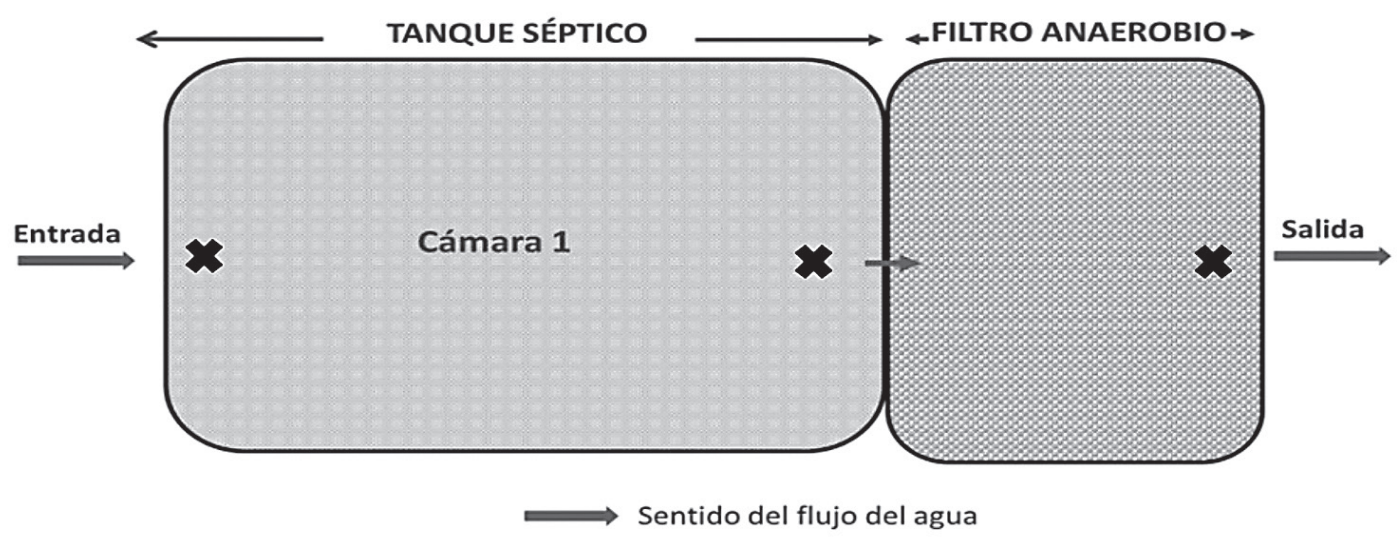

Figura 2. Puntos de muestreo del tanque séptico de los Apartamentos.

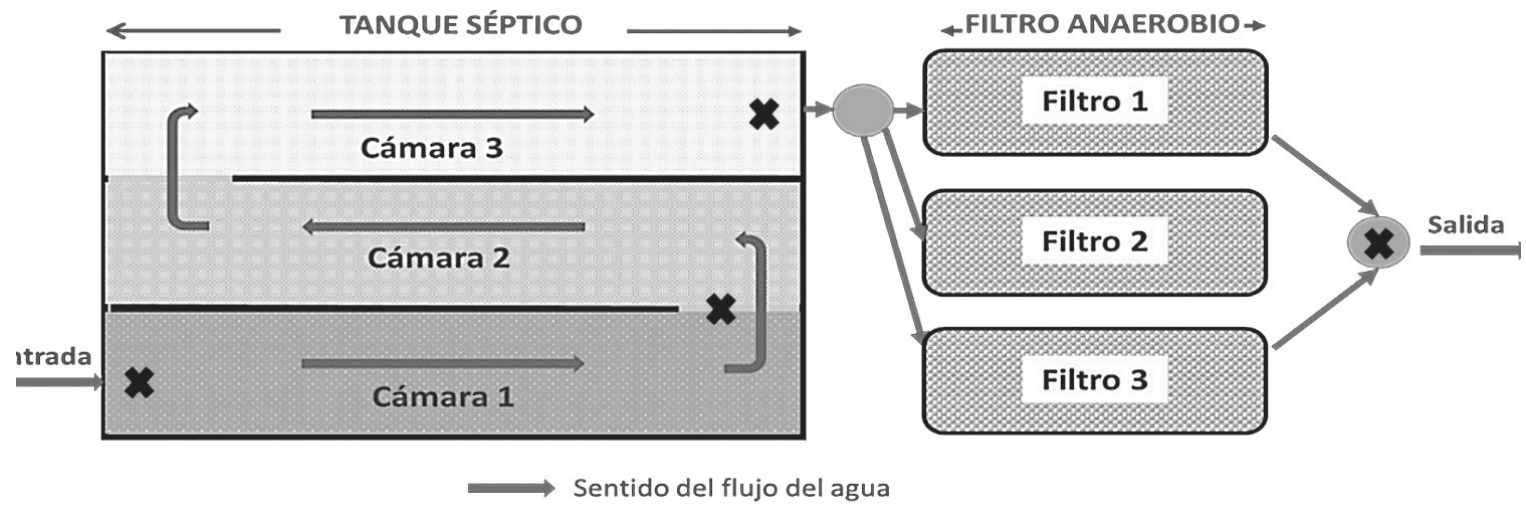

Figura 3. Puntos de muestreo del sistema de tratamiento del Condominios.

En el sistema de tratamiento de la Casa fueron seleccionados 4 diferentes sitios para la caracterización del agua residual, uno en la entrada del tanque séptico, otros dos en las salidas de cada cámara y por último, en la salida del FAFA (figura 4).

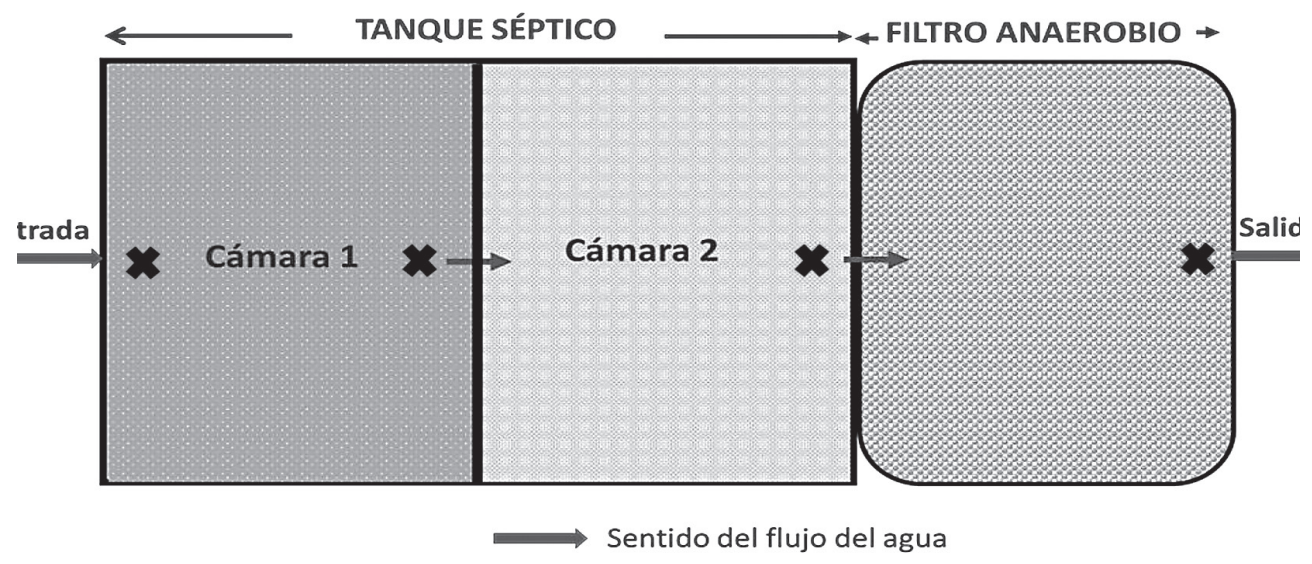

Figura 4. Puntos de muestreo del sistema de tratamiento de la Casa de Escazú. 


\section{Resultados y Discusión}

\section{Aguas residuales de Apartamentos}

La figura 5 muestra las variaciones de caudal a lo largo de un día entre semana (25 de noviembre 2015) bajo condiciones normales de rutina de los habitantes. Se presentó un incremento en el caudal entre las 6:45 a.m. y las 9:00 a.m. siendo la hora pico las 7:15 a.m., teniendo como punto máximo un caudal de $0.07 \mathrm{~L} / \mathrm{s}$. Se presentó un leve incremento en horas del medio día; mientras en periodos entre la 1:00 p.m. y las 5:00 p.m. no hubo generación de aguas residuales. En este último período los habitantes probablemente estuvieron en actividades que no implican el uso del agua o están fuera de la residencia y cuyo retorno ocurrió después de las 5:30 p.m.; retomándose la producción de aguas residuales la cual usualmente se extiende hasta las 11:30 p.m. Las actividades de lavandería se registran en su mayoría, en horas de la noche ya que la calidad del afluente presenta mayor cantidad de espuma o agua jabonosa.

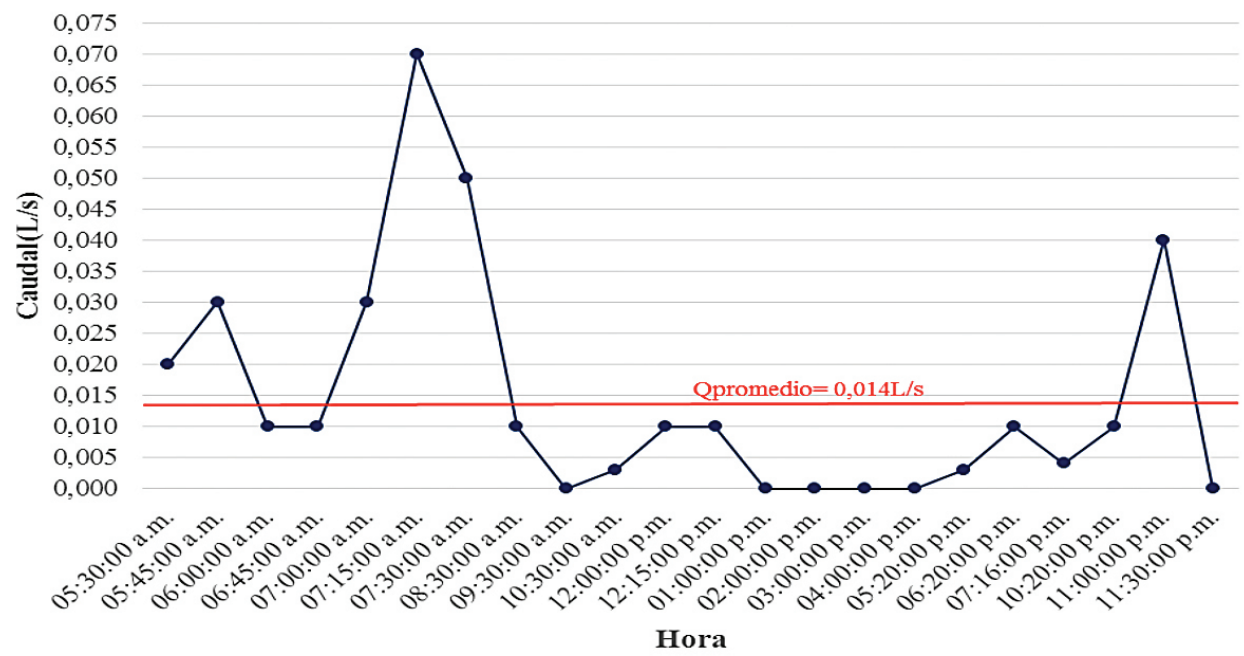

Figura 5. Producción de aguas residuales en Apartamentos.

El caudal promedio fue de $0.014 \mathrm{~L} / \mathrm{s}$ con una desviación estándar de 0.019 y que varían en un rango entre un mínimo de 0.000L/s y un máximo de 0.070L/s. El pH del agua residual varió entre 6.66 y 7.28 con un promedio de $7.01 \pm 0.24$; las temperaturas se mantuvieron en un rango entre $21.3^{\circ} \mathrm{C}$ y $25.7^{\circ} \mathrm{C}$ con un promedio de $(24.0 \pm 1.0)^{\circ} \mathrm{C}$. La figura 6 muestra la variación del contenido de $\mathrm{DBO}_{5}$ en cada una de las etapas de un tanque séptico instalado en los Apartamentos. Durante el período de estudio, el valor promedio de $\mathrm{DBO}_{5}$ medido en la entrada al tanque séptico fue de $451 \mathrm{mg}$ de $\mathrm{O}_{2} / \mathrm{L}$, mientras que el valor a la salida del tanque fue de $403 \mathrm{mg} / \mathrm{L}$. Esta diferencia corresponde a un 10,7\% de remoción de la materia orgánica. Por otra parte, el contenido de $\mathrm{DBO}_{5}$ a la salida del FAFA fue de $285 \mathrm{mg}$ de $\mathrm{O}_{2} / \mathrm{L}$. La reducción de materia orgánica dentro del FAFA correspondió a un 29,3\% y la disminución total considerando ambas etapas fue de un 36,9\%.

En el caso de los SST, los niveles de remoción fueron mayores; el contenido promedio de SST a la entrada del tanque fue $375 \mathrm{mg} / \mathrm{L}$ y a la salida del tanque fue de $152 \mathrm{mg} / \mathrm{L}$, lo que representa una remoción de un 59,6\%. Los niveles de SST a la salida del FAFA fueron de $64 \mathrm{mg} / \mathrm{L}$, que corresponde a una disminución de un $57,5 \%$ en esta etapa. Si se consideran ambos procesos, entonces la reducción total fue de un 82,8\% (figura 7). 


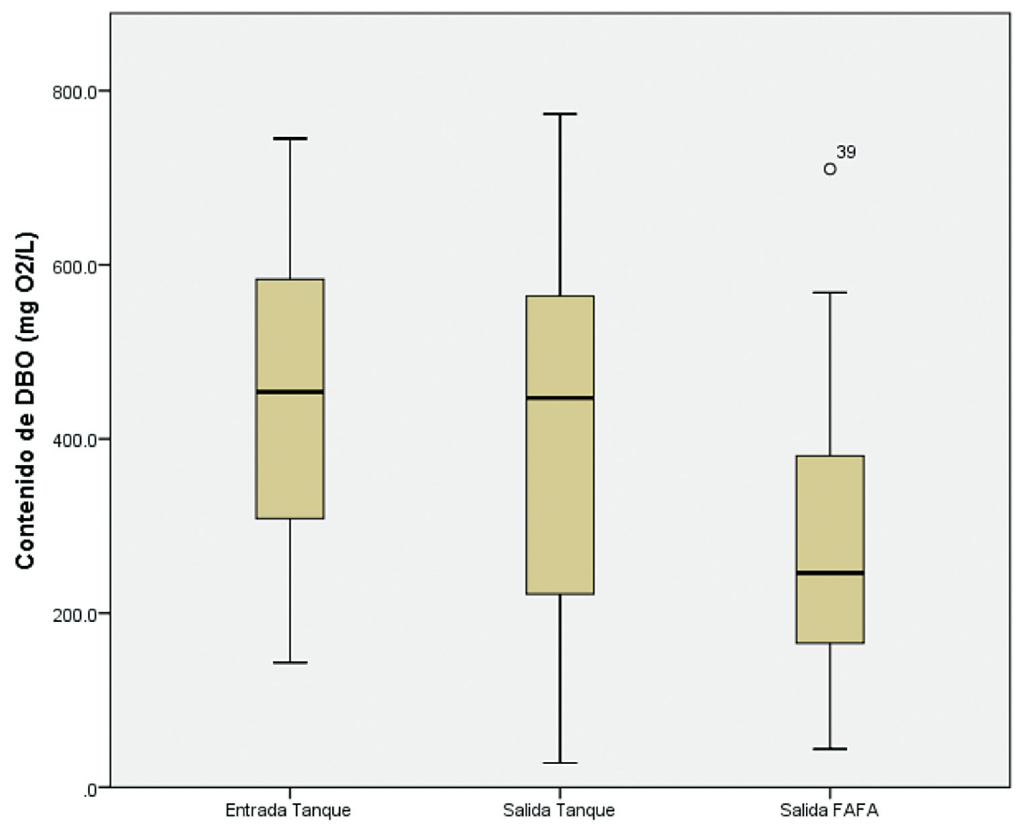

Figura 6. Variación del contenido de $\mathrm{DBO}_{5}$ en cada etapa del tanque séptico.

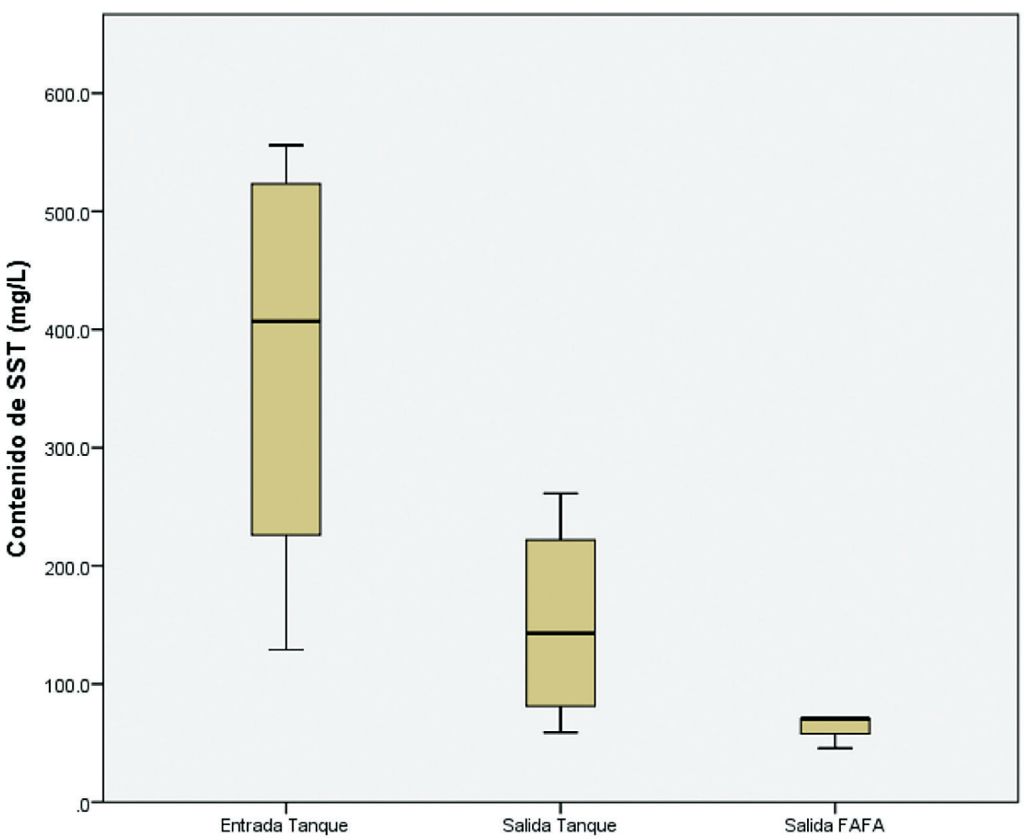

Figura 7. Variación del contenido de SST en cada etapa del tanque séptico

En términos generales los parámetros $\mathrm{DBO}_{5}$ y SST de las aguas residuales son muy variables y sensibles a los cambios de las actividades domésticas y no guardan relación directa con la hora del día o el día de muestreo (figuras 6 y 7). A pesar de las fluctuaciones en la calidad del agua residual, los parámetros de $\mathrm{pH}$ y temperatura sí muestran valores muy estables alrededor de 7.01 y $24.0^{\circ} \mathrm{C}$, respectivamente. 


\section{Aguas residuales de Condominios}

La figura 8 presenta el comportamiento de la producción de aguas residuales en el Condominios a lo largo de un día entre semana (10 de diciembre 2015) bajo condiciones normales de rutina de los habitantes del Condominios. El comportamiento es variable con valores más altos durante la mañana mostrando los valores mayores entre las 8:30 a.m. y las 10:30 a.m.; por otra parte, el caudal más alto de $0.180 \mathrm{~L} / \mathrm{s}$ se registró a las 9:00 a.m. Durante el transcurso de la tarde (de 1:30 p.m. hasta las 6:00 p.m.) se observaron caudales de entrada muy bajos; esto debido a que la actividad de limpieza disminuyó y por lo general, son realizadas por un personal externo durante las horas de la mañana y hasta el mediodía. La mayoría de los habitantes de cada apartamento regresa después de las 6:00 de la tarde y no se evidenció producción de aguas residuales a partir de las $7 \mathrm{pm}$.

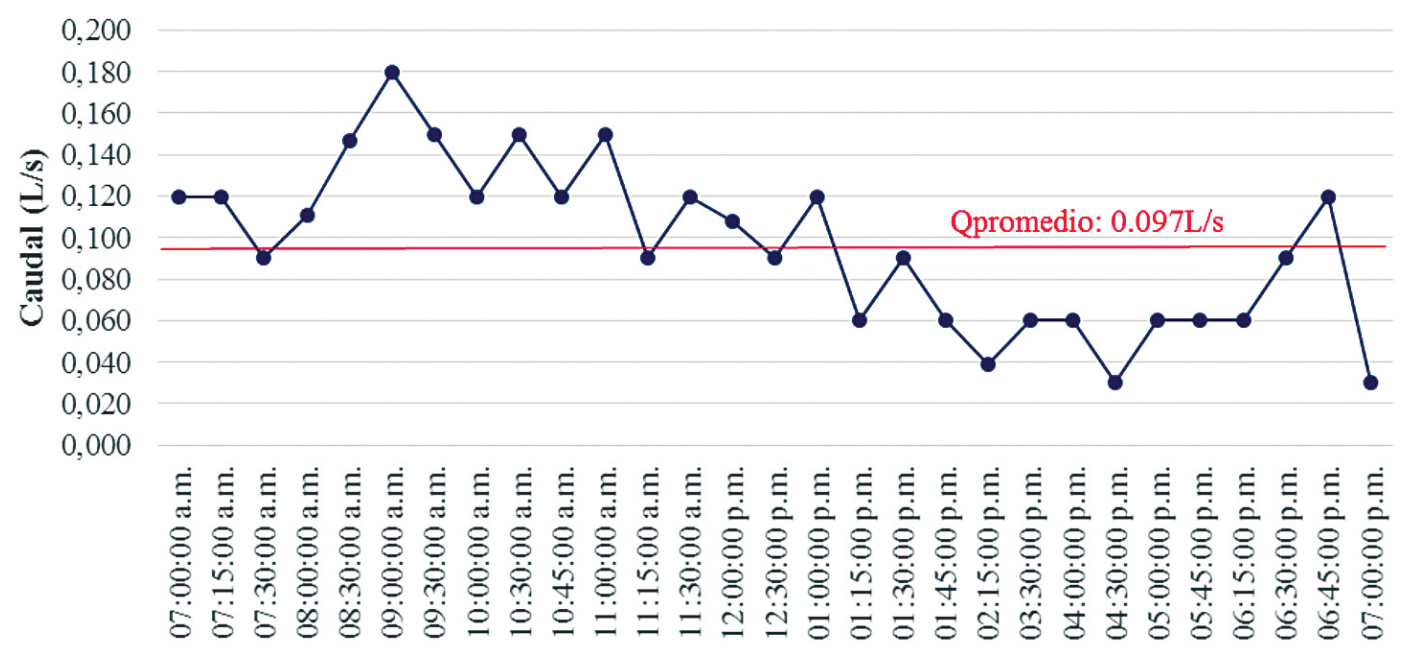

Hora

Figura 8. Producción de aguas residuales en Condominios.

El caudal promedio obtenido fue de $0.097 \mathrm{~L} / \mathrm{s}$ con una desviación de 0.040 y varió entre un rango mínimo de $0.030 \mathrm{~L} / \mathrm{s}$ hasta un máximo de $0.180 \mathrm{~L} / \mathrm{s}$. La temperatura del agua residual osciló en un rango entre $25.4^{\circ} \mathrm{C}$ y $27.2^{\circ} \mathrm{C}$ con un valor promedio de $(26.0 \pm 0.5)$, mientras que el $\mathrm{pH}$ varió entre (6.80-7.50) con un promedio de (7.19 \pm 0.25$)$.

La figura 9 muestra las variaciones del $\mathrm{DBO}_{5}$ medidas en cada etapa del sistema de tratamiento instalado en los Condominios. El valor promedio de $\mathrm{DBO}_{5}$ de entrada fue de $357 \mathrm{mg}$ de $\mathrm{O}_{2} / \mathrm{L}$ y a la salida del tanque séptico fue de 226, lo que representa una remoción de 36,5\%. Por otra parte, el valor promedio de salida del FAFA fue de $191 \mathrm{mg}$ de $\mathrm{O}_{2} / \mathrm{L}$, lo que corresponde a una remoción de 15,7 en esta segunda etapa. Si se considera la remoción global desde la entrada al tanque hasta la salida del FAFA, la remoción fue de $46,5 \%$.

El caso de los SST en los condominios se muestra en la figura 10 donde el valor promedio de entrada de SST fue de $450 \mathrm{mg} / \mathrm{L}$ y el de salida en el tanque fue de $88 \mathrm{mg} / \mathrm{L}$, lo que representa una remoción de un 80,6\%. El valor promedio de salida del FAFA fue de $73 \mathrm{mg} / \mathrm{L}$, lo que corresponde a una remoción de un $16,6 \%$ si se considera el valor de ingreso dentro del FAFA. Por otra parte, si evaluamos este valor de salida del FAFA con respecto al valor de entrada al tanque, la remoción correspondió a un 83,8\%. 


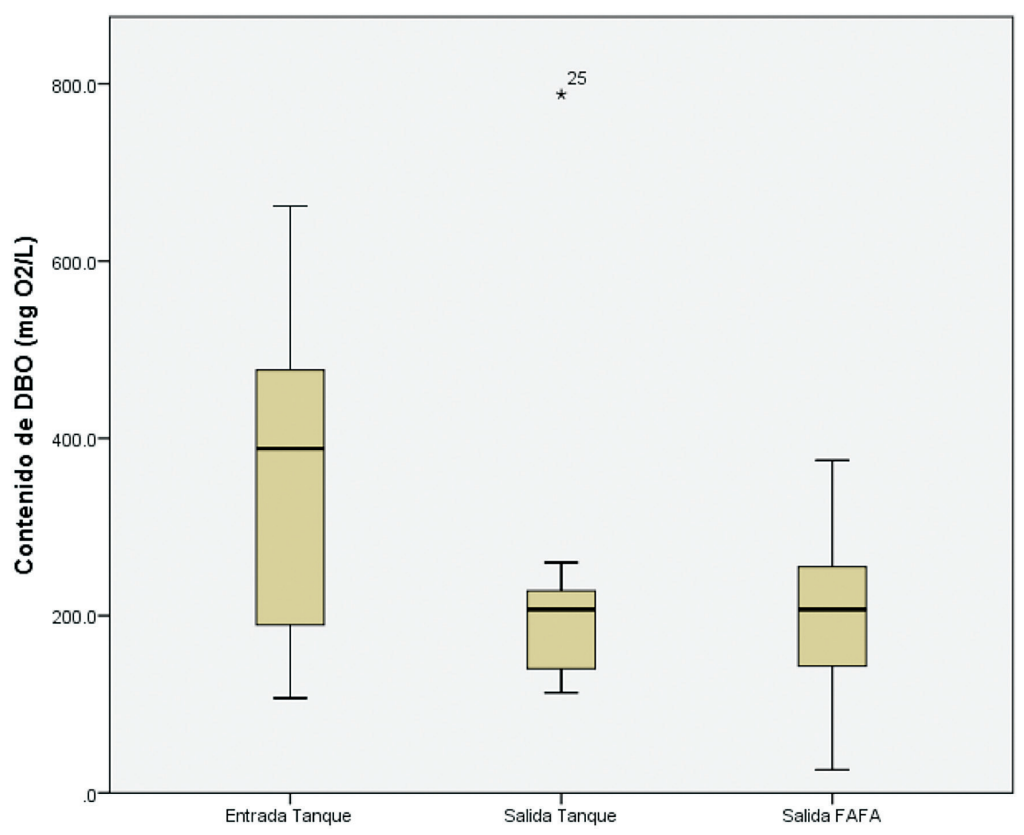

Figura 9. Variación del contenido de $\mathrm{DBO}_{5}$ en cada etapa en el multifamiliar Condominios.

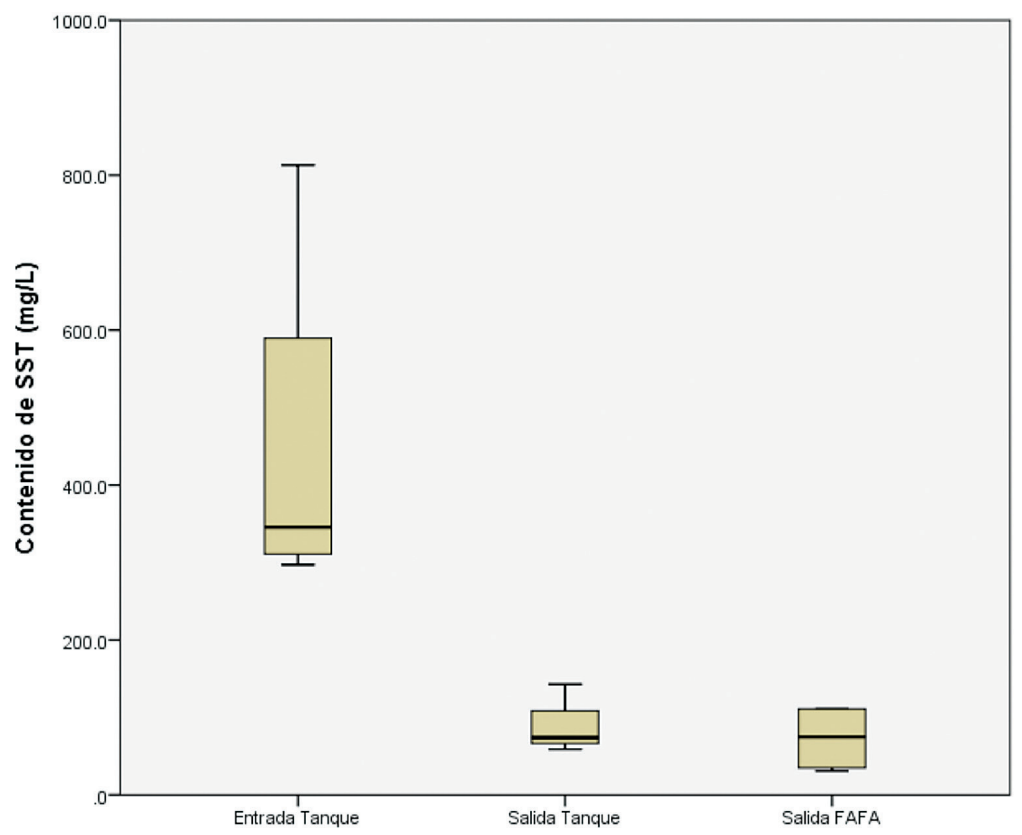

Figura 10. Variación del contenido de SST en cada etapa en el multifamiliar Condominios.

No se pudo establecer una relación entre la concentración de la $\mathrm{DBO}_{5}$ y los SST ya que en todos los muestreos el comportamiento de los resultados fue muy variable, tanto a lo largo del día como entre los días de muestreo. Solamente los SST a las 2:00 p.m. tienen una desviación estándar baja mientras que para los demás resultados tanto en $\mathrm{DBO}_{5}$ como en SST, existen desviaciones estándar altas. En ambos parámetros también puede observarse que los valores máximos se alcanzan entre las 8:00 a.m. y las 10:00 a.m. y disminuyen en el transcurso del día, mostrando el punto más bajo a las 2:00 p.m. 


\section{Aguas residuales de Casa}

La Casa se caracterizó por pasar desocupada la mayor parte del día lo que imposibilitó llevar a cabo un análisis de caudal en forma horaria. Por lo tanto, los muestreos realizados fueron puntuales con una frecuencia mensual en las primeras horas de la mañana las cuales fueron las consideradas como las horas pico en la generación de aguas residuales.

La temperatura para el agua residual varió entre $21.4^{\circ} \mathrm{C}$ y $22.4^{\circ} \mathrm{C}$, con un promedio de $22.0^{\circ} \mathrm{C}$ $\pm 0.6^{\circ} \mathrm{C}$, mientras que el $\mathrm{pH}$ tuvo un rango entre 7.48 y 8.14 y un promedio de $7.76 \pm 0.34$. La figura 11 muestra las variaciones de $\mathrm{DBO}_{5}$ en distintas etapas del tanque séptico instalado en la

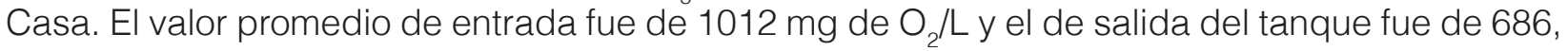
lo que representa una remoción de un 32,2\%. El valor promedio de salida del FAFA fue de 738 $\mathrm{mg}$ de $\mathrm{O}_{2} / \mathrm{L}$. Si se compara este valor con el de salida del tanque, corresponde a una remoción negativa indicando que este proceso fue ineficiente. Al comparar el valor promedio de entrada al tanque con el de salida del FAFA se obtuvo una remoción de un 27,1\%.

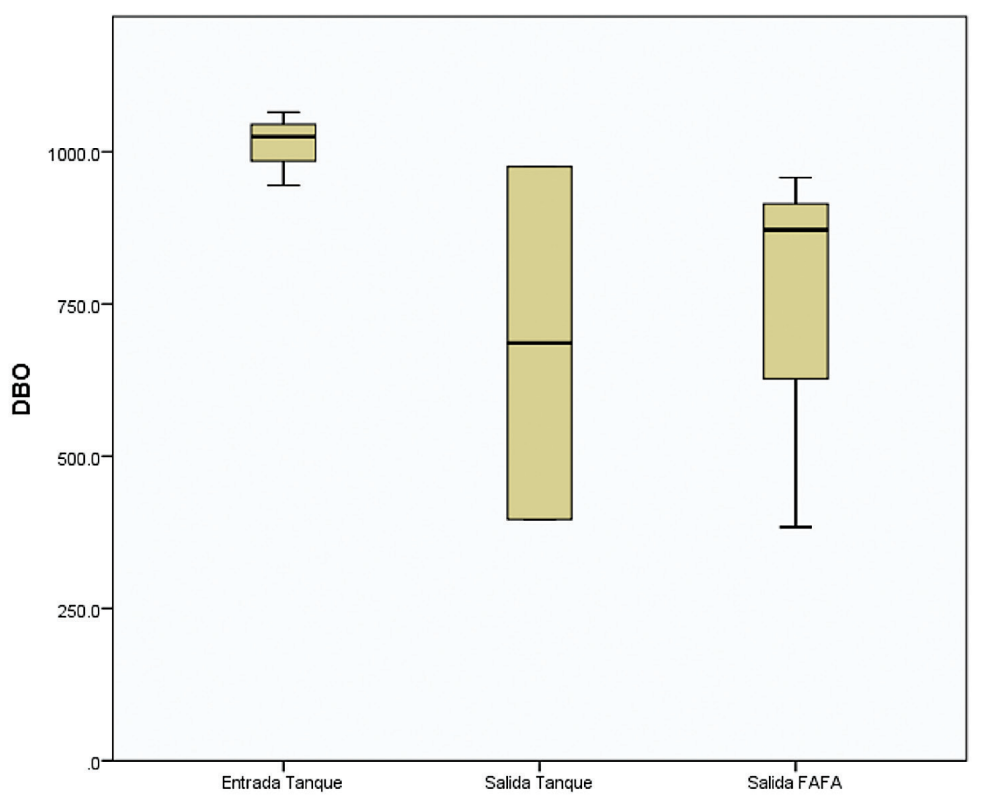

Figura 11. Variación del contenido de $\mathrm{DBO}_{5}$ en cada del sistema de tratamiento en Casas.

El contenido promedio de SST a la entrada del tanque en la Casa fue $1009 \mathrm{mg} / \mathrm{L}$ y a la salida del tanque fue de $72 \mathrm{mg} / \mathrm{L}$, lo que representa una remoción de un 92,9\%. Los niveles de SST a la salida del FAFA fueron de $36 \mathrm{mg} / \mathrm{L}$, que corresponde a una disminución de un 50,0\% en esta etapa. Si se consideran ambos procesos, entonces la reducción total fue de un 96,4\% (figura 12).

La calidad del agua residual en Casa es muy cambiante en las concentraciones en los parámetros $\mathrm{DBO}_{5}$ y SST ya que, al tener caudales de entrada tan pequeños, una descarga que se haga puede marcar la diferencia [13].

En el caso de los valores de aguas residuales domésticas en Apartamentos y Condominios, los parámetros de pH, temperatura, DBO5 y SST se encuentran entre los rangos teóricos. En el caso de la Casa el valor en las concentraciones de $\mathrm{DBO}_{5}$ y de SST son mayores, dado que el agua residual de entrada no se encuentra diluida, por recibir solamente aguas de los inodoros; mientras que en los otros dos sitios, se encuentran mezcladas las aguas pardas con 
las aguas grises; provenientes de los diferentes usos de duchas, servicios sanitarios, lavadoras, lavaplatos y otros generadores de aguas residuales. Además, el flujo de entrada al sistema de la casa es menor comparado con los otros dos sistemas en estudio.

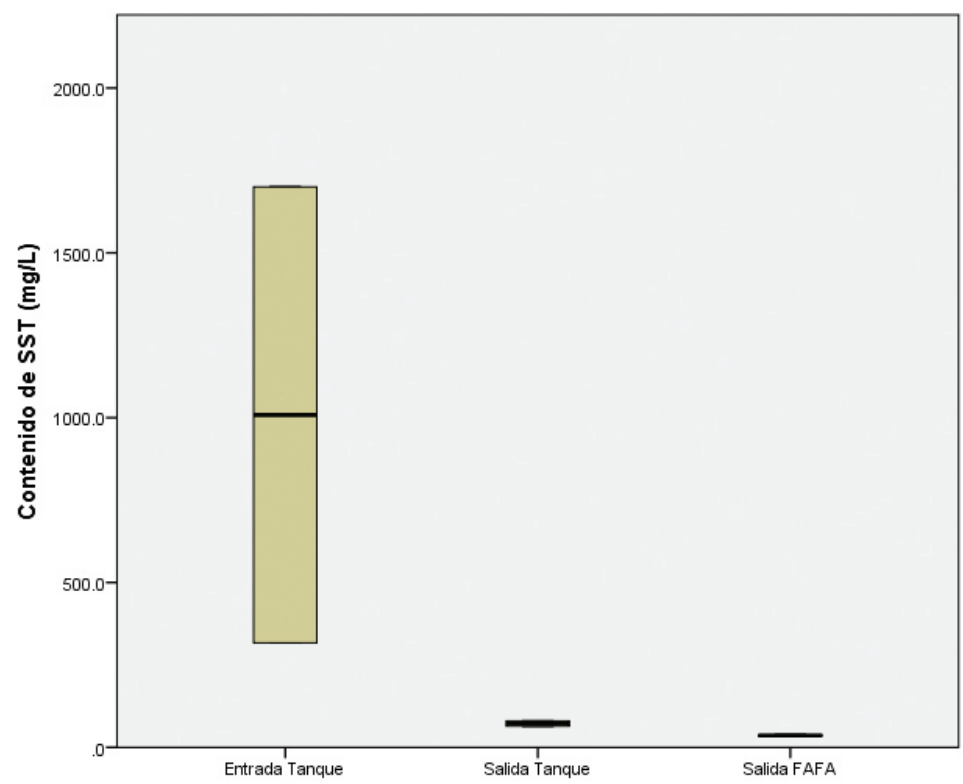

Figura 12. Variación del contenido de SST en cada etapa en la vivienda unifamiliar Casa.

Respecto a la temperatura, no se tiene un rango claramente establecido para las aguas residuales, ya que éste varía según la temperatura ambiente de cada lugar. Los resultados obtenidos para todos los sistemas de tratamiento son similares, y se acoplan a temperaturas de la zona en la que están ubicados; donde según el Instituto Meteorológico Nacional, la temperatura ambiente promedio anual es de $20.8^{\circ} \mathrm{C}$ en la zona de Escazú. Las temperaturas del agua residual obtenidas son propias de afluentes en países tropicales las cuales tienen ventajas en el funcionamiento por su estabilidad climática, lo cual permite un crecimiento y proliferación estable de la vida microbiana; facilitando el proceso de las reacciones químicas y manteniendo normales las velocidades de reacción [8]. Los valores de pH son igualmente beneficiosos para el desarrollo de los procesos biológicos; por lo que no requieren ningún tipo de mecanismo de neutralización de pH para desempeñar adecuadamente la depuración de aguas residuales por parte de los microorganismos [14].

Al considerar todos los parámetros físicos, químicos y biológicos evaluados, se puede confirmarse la teoría de Méndez et al. [13], donde se menciona que la calidad de agua residual de una sistema de tratamiento individual en aguas domésticas es muy variante respecto al tiempo, por presentar caudales pequeños los cuales cambian también horariamente y según al uso del agua dentro de los hogares, en particular para viviendas unifamiliares o multifamiliares de pocas unidades de vivienda, donde no hay amortiguación o regulación de las cargas.

\section{Evaluación de los sistemas tanque séptico-y filtro anaerobio}

En el lugar Apartamentos, tanto los SST como la DBO5 tienden a disminuir después de cada etapa de tratamiento, aunque el valor de la variable a la salida no es un valor constante pues parece estar asociada a cambios en carga contaminante de entrada, variaciones en los caudales y los tiempos de retención de la masa de agua en función del tiempo. En términos de SST, hay 
una disminución notable en el tanque séptico y menos marcada en el FAFA, lo cual destaca la labor efectuada por el tanque séptico en la remoción de los SST. Caso contrario ocurre con la $\mathrm{DBO}_{5}$ ya que se da una baja remoción en el tanque séptico mientras que es más significativa en el FAFA. Por otra parte, el sistema se encuentra limitado debido a las altas concentraciones de $\mathrm{DBO}_{5}$ y no genera efluentes que cumplan la normatividad de Costa Rica que es $50 \mathrm{mg} / \mathrm{L}$ tanto para $\mathrm{DBO}_{5}$ como SST, en el caso de que estos efluentes sean descargados a un cuerpo receptor.

En el caso de Condominios, la principal remoción de SST se da en la cámara 1 haciéndose menos notoria en el paso de cada una de las cámaras. La remoción en el $\mathrm{DBO}_{5}$ fue notoria en la cámara 1 aunque menor a la salida de la cámara 3, siguiendo con una remoción después del paso por el FAFA. En este sistema, la mayor reducción de $\mathrm{DBO}_{5}$ se llevó a cabo en la cámara 1 del tanque séptico. CEPIS [9] menciona que la compartimentalización de los tanques sépticos se favorece que en el primer compartimento se dé una mayor sedimentación y digestión de las partículas del agua residual.

Con respecto al desempeño del sistema en Casa, tanto las concentraciones de SST como de $\mathrm{DBO}_{5}$ disminuyen conforme van avanzando en el proceso presentando las diferencias más notorias en la primera cámara. Para ambos parámetros, no hay una baja significativa entre el efluente del tanque séptico y la salida del FAFA.

En general, se observó una remoción de la carga de SST y $\mathrm{DBO}_{5}$ en cada fase del tratamiento, siendo esta más notoria en la primera cámara del tanque séptico, debido a los procesos físicos y biológicos, propios de esta unidad, que propician tiempos de retención hidráulicos mayores para la sedimentación de los sólidos y el consumo de la materia orgánica por parte de los microorganismos [8]. En el tanque séptico se da una remoción significativa de SST y en menor escala de $\mathrm{DBO}_{5}$, mientras en el FAFA la remoción de SST es más baja ya que el material particulado en su afluente es menor, propiciándose la remoción de $\mathrm{DBO}_{5}$ por la densidad microbiana que se da en la biopelícula formada en el lecho filtrante [10].

La variación de los caudales asociados a los diferentes usos del agua y dinámicas de los habitantes, afectan los tiempos de retención, teniendo en cuenta que hay masas de agua que se someten a largos tiempos de residencia dado los periodos donde no hay producción de aguas residuales como las tardes o las noches o tiempos de retención más cortos asociados a los picos de caudal como los que se presentan en la hora de la mañana o el medio día [13].

\section{Conclusiones}

En los sitios evaluados, los parámetros $\mathrm{DBO}_{5}$ y SST, indicadores de calidad de agua residual doméstica en viviendas unifamilares o multifamiliares con poca cantidad de unidades de vivienda, son muy fluctuantes en relación con la hora del día muestreado, así como el día de monitoreo. Se presentaron rangos amplios en sus comportamientos y desviaciones altas, lo cual está asociado con los diferentes caudales producidos como consecuencia de los diferentes usos y prácticas del agua que se dan en los hogares. Por lo tanto, no se puedo definir una tendencia de comportamiento según la hora del día; sin embargo, los parámetros pH y temperatura son muy estables, tanto a lo largo del día como en los diferentes días.

Variaciones en las actividades domésticas en el tiempo generan a su vez, variaciones en los caudales y las calidades de aguas residuales. Dependiendo de la hora del día, se observan aguas residuales con características de variable carga tanto en $\mathrm{DBO}_{5}$ como SST.

La evaluación físicoquímica y biológica, indica que los sistemas compuestos por tanques sépticos son muy sensibles y dependientes de las concentraciones de contaminantes en la 
entrada y los tiempos de retención variables que se presentan, las cuales van a estar asociados a las variaciones horarias; por lo cual no se puede definir adecuadamente porcentajes de eficiencia en la remoción de las cargas contaminantes.

$\mathrm{El} \mathrm{pH}$ a lo largo del tren de tratamiento es muy estable, con una leve tendencia a la baja, debido a la digestión anaerobia de materia orgánica y por ende la producción de ácidos grasos volátiles, generados por los microorganismos del tanque séptico y el filtro anaerobio de flujo ascendente.

En el sistema de tratamiento de Apartamentos, el cual es un sistema integrado sin compartimentos; se obtiene que la mayor remoción de sólidos suspendidos totales se da en el tanque séptico, mientras que la mayor remoción de $\mathrm{DBO}_{5}$ tiene lugar al FAFA que actúa como tratamiento secundario.

En los sistemas de tratamiento compartamentalizados usados en Condominios y Casa, presenta la mayor remoción de SST y $\mathrm{DBO}_{5}$ en la primera cámara. En general, se presenta la disminución de los valores de estos parámetros conforme el flujo de agua avanza a lo largo del sistema, desde la entrada del tanque séptico hasta la salida del filtro anaerobio de flujo ascendente.

La acumulación de lodos se valoró en dos periodos mensuales diferentes, obteniendo resultados muy diferentes tanto en los diferentes compartimentos como a lo largo del tiempo; por lo que se concluye que la cantidad y calidad del lodo es variable a través del tiempo y dependiendo además de la calidad de uso del agua. Prácticas como la colocación del papel higiénico en el sanitario, genera un incremento notable en la producción de lodos.

\section{Agradecimientos}

Los autores expresan sus agradecimientos al Instituto Tecnológico de Costa Rica (ITCR). También, agradecemos al Centro de Investigación en Protección Ambiental (CIPA) y al Laboratorio de Servicios Químicos y Microbiológicos (CEQIATEC) por su gran respaldo a esta investigación.

\section{Referencias}

[1] E. Rosales, Tanques sépticos. Conceptos teóricos base y aplicaciones. Tecnología en Marcha vol. 18, n.o 2, pp. 26-33, 2005. Recuperado de http://revistas.tec.ac.cr/index.php/tec_marcha/article/view/205

[2] F. Ruiz, Gestión de las excretas y aguas residuales en Costa Rica: situación actual y perspectiva. 2012. Recuperado de https://www.aya.go.cr/centroDocumetacion/catalogoGeneral/Gesti\%C3\%B3n\%20de\%20 las\%20Excretas\%20y\%20Aguas\%20Residuales\%20en\%20Costa\%20Rica\%20\%20Situaci\%C3\%B3n\%20 Actual\%20y\%20Perspectiva.pdf

[3] S. Simón, Determinación De La Concentración De Nitrógeno En Lodos Activados Para Su Uso Como Biofertilizante (Tesis de ingeniería). Universidad Autónoma Agraria Antonio Narro, Torreón, México, 2015. Recuperado de http://repositorio.uaaan.mx:8080/xmlui/handle/123456789/7909

[4] E. López, J.G. Martínez, y J.L. García, Determinación de Sólidos suspendidos totales y Sólidos Suspendidos Volátiles. 2014. Recuperado de http://www.academia.edu/9209870/Determinaci\%C3\%B3n_ de_S\%C3\%B3lidos_Suspendidos_Totales_SST_y_S\%C3\%B3lidos_Suspendidos_Volatiles_SSV_en_agua_tratada

[5] A. Pérez y P. Torres, Evaluación del comportamiento hidrodinámico como herramienta para optimización de reactores anaerobios de crecimiento en medio fijo. Rev. Fac. Ing. Univ. Antioquia, vol. 45, pp. 27-40, 2008. Recuperado de http://www.scielo.org.co/pdf/rfiua/n45/n45a03.pdf

[6] P. Torres, Perspectivas del Tratamiento Anaerobios de Aguas Residuales Domésticas en Países en Desarrollo. Revista ElA, vol. 18, pp. 115-129, 2012. Recolectado de http://www.redalyc.org/html/1492/149225098009/ 
[7] D. Mara, Domestic Wastewater Treatment in Developing Countries. London, UK: Earthscan. 2003. Recuperado de https://www.pseau.org/outils/ouvrages/earthscan_Itd_domestic_wastewater_treatment_in_developing_ countries_2003.pdf

[8] D. Mara y G.S. Sinnatamby, Rational design of septic tanks in warms climates. The Public Health Engineer, vol. 14, no. 4, pp. 49-55, 1986.

[9] Centro Panamericano de Ingeniería Sanitaria y Ciencias del Ambiente, Guía para el diseño de tanques sépticos, tanques imhoff y lagunas de estabilización. 2003. Recuperado de http://www.bvsde.paho.org/tecapro/ documentos/sanea/163esp-diseno-ti.pdf

[10] R. Busato y U. Palowsky, Estudo Cinético De Remoção De Matéria Orgânica De Um Filtro Anaeróbio De Fluxo Ascendente Usado Como Tratamento De Efluente De Reator Uasb: Estudo De Caso Da Ete De Imbituva. Curitiba, Brasil. 2005. Recuperado de http://repositorio.roca.utfpr.edu.br/jspui/handle/1/4976

[11] T. Quiterio, Tratamiento de Agua Residual Doméstica Mediante el Uso de un Filtro Anaerobio Empacado con Espuma de Poliuretano a Diferentes Tiempos de Retención Hidráulica (Tesis de ingeniería). Universidad Autónoma Agraria Antonio Narro, Coahuila, México. 2014. Recuperado de http://repositorio.uaaan.mx:8080/ xmlui/handle/123456789/6496

[12] A. D. Eaton, L.S. Clesceri, A. E. Greenberg y M.A.H. Franson, Standard methods for the examination of water and wastewater. 22nd ed. Washington, DC: American Public Health Association. 2012.

[13] R. Méndez, A. Guijón y C. Quintal, Determinación de la tasa de acumulación de lodos en fosas sépticas de la ciudad de Mérida, Yucatán. Ingeniería, vol. 11, no. 3, pp. 55-64, 2007. Recuperado de http://www.revista. ingenieria.uady.mx/volumen11/determinacion_de_la_tasa.pdf

[14] Metcalf \& Eddy, Inc., Características de las aguas residuales. En: Autor (eds.). Ingeniería de aguas residuales: Tratamiento, vertido y reutilización, pp. 53-135, 1995. Madrid, España: McGraw Hill. Recuperado de http://aulacidta5.usal.es/Bibliocidta/FMPro?-db=bibliocidta.fp3\&-format=record_detail.htm\&lay=presenta1\&Materia=Tratamiento\%20de\%20aguas\&-max=20\&-recid=33\&-find y http://cidta.usal.es/cursos/ETAP/modulos/libros/Caracteristicas.PDF 